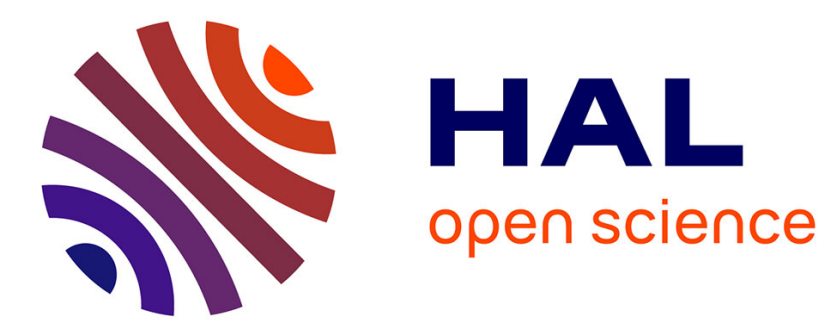

\title{
Drag fluctuations of a disk in a turbulent jet: effect of turbulent scales averaging
}

Bertrand Thiria, Olivier Cadot, Jean-François Beaudoin

\section{To cite this version:}

Bertrand Thiria, Olivier Cadot, Jean-François Beaudoin. Drag fluctuations of a disk in a turbulent jet: effect of turbulent scales averaging. EPL - Europhysics Letters, 2009, 87 pp.44007. 10.1209/02955075/87/44007 . hal-01289958

\section{HAL Id: hal-01289958 \\ https://hal-ensta-paris.archives-ouvertes.fr/hal-01289958}

Submitted on 26 Nov 2017

HAL is a multi-disciplinary open access archive for the deposit and dissemination of scientific research documents, whether they are published or not. The documents may come from teaching and research institutions in France or abroad, or from public or private research centers.
L'archive ouverte pluridisciplinaire HAL, est destinée au dépôt et à la diffusion de documents scientifiques de niveau recherche, publiés ou non, émanant des établissements d'enseignement et de recherche français ou étrangers, des laboratoires publics ou privés. 


\title{
Drag fluctuations of a disk in a turbulent jet: Effect of turbulent scales averaging
}

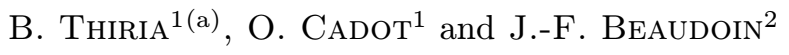 \\ ${ }^{1}$ Unité de Mécanique, École supérieure de Techniques avancées - Chemin de la Hunière, 91761 Palaiseau Cedex, \\ France, EU \\ ${ }^{2}$ Department of Research and Innovation, PSA Peugeot-Citroen - 2 route de Gisy, 78943 Vélizy-Villacoublay, \\ France, EU
}

\begin{abstract}
The drag fluctuations of a disk placed on the axis of a turbulent incompressible jet are studied at $R e=70000$. Statistics and spectra have been obtained for different disk sizes. A significant spatial averaging effect is observed in the symmetrization of probability distribution function and in the low-pass filtering of spectra. These effects are associated with a redistribution of the high-frequencies energy to the low frequencies. It is shown that this redistribution is done in such a way that the rms value of the drag fluctuations increases linearly with the disk surface. These results concerning the drag fluctuations are compared and found to be consistent with fluctuations of a global kinetic energy extracted from the turbulent field in front of the disk.
\end{abstract}

Introduction. - Fluctuations of global quantities in out-of-equilibrium systems are of general interest. For the last ten years, a global quantity as the power injected in a closed turbulent flow has been thoroughly studied [1-7]. The fluctuations are not symmetric $[1,8]$ but tend to symmetrize as the degrees of freedom are increased $[5,8,9]$. In the closed flow, the number of degrees of freedom strongly depends on the aspect ratio of the experiment that determines the number of large-scale structures $[5,9]$. Hence, the central limit theorem, implying a symmetrization of the fluctuations together with a reduction of the fluctuation rate as the number of degrees of freedom is increased is satisfactorily verified [5].

There are far fewer experimental results concerning the case of open turbulent flows which are also of general interest for direct industrial applications. For instance, questions of practical importance are: how do the global force fluctuations exerted on a body under turbulent conditions behave with different body sizes and flow characteristics? Is there a smoothing effect of the fluctuations due to a spatial averaging of the turbulent scales? Is the central

\footnotetext{
(a) Present address: Physique et Mécanique des Milieux Hétérogènes (PMMH) UMR 7636 CNRS ESPCI P6 P7 - 10 rue Vauquelin, F-75231, Paris Cedex 5, France, EU; E-mail: bthiria@pmmh .espci.fr
}

limit theorem relevant for this case? For open turbulent flows, we are actually only aware of the work of Ciliberto et al. [10] who considered the drag fluctuations of a disk placed in a fully turbulent jet. The authors were studying the temporal averaging of the drag fluctuations in order to bring some experimental evidence of the Gallavotti-Cohen fluctuation theorem (see references in [10]). This theorem predicts the ratio of the probabilities for positive- and negative-energy injection rates (a quantity related to the instantaneous drag) averaged over a variable time window. In order to observe negative events of drag (an essential ingredient for the fluctuation theorem), the authors placed the disk in a very specific region, not on the axis of the turbulent jet where the instantaneous drag is always positive (as we will do in this work), but sufficiently far from the axis to obtain some negative drag events. While the work of Ciliberto et al. [10] was a temporal averaging study, the goal of the present work is a spatial averaging study of the turbulent scales impacting on the disk surface.

We considered two cases in a turbulent jet flow at a fixed Reynolds number. In the first case, the global quantity is directly the drag that the turbulent jet exerts on a disk of diameter $D$. In the second case, the global quantity is a kinetic energy contained in a square area in front of the disk. The aim of this work is to study effects of 


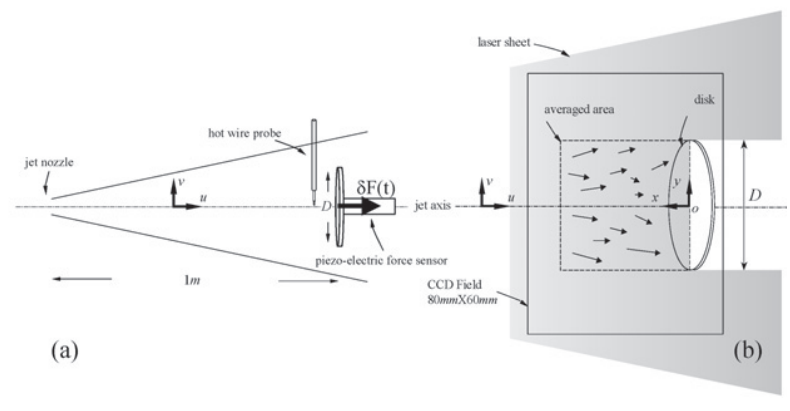

Fig. 1: Experimental setup. (a) Setup for drag and local velocity measurements. (b) PIV measurements setup with the disk having the larger size $D=40 \mathrm{~mm}$. The camera field dimensions are fixed, while the size of the averaged area (see text) displayed by a dashed line is adapted to the disk diameter.

the spatial averaging of the turbulent scales on the drag fluctuations of the disk. The question we address is: what are the properties of these drag fluctuations as the disk size increases?

Experimental setup. - The turbulent air jet is formed by a nozzle of $20 \mathrm{~mm}$ in diameter. We used five disks having diameters of $10 \mathrm{~mm}, 15 \mathrm{~mm}, 22 \mathrm{~mm}, 30 \mathrm{~mm}$ and $40 \mathrm{~mm}$. Each disk was successively placed one meter from the nozzle, centred on the jet axis and perpendicular to it (fig. 1). At this location and without disk, the freejet width is $\delta=110 \mathrm{~mm}$, the mean velocity taken on the jet axis is $\langle u\rangle=10 \mathrm{~m} \cdot \mathrm{s}^{-1}$, the fluctuation rate defined as $\frac{u_{r m s}}{\langle u\rangle}$ is $28 \%$, and the corresponding Reynolds number based on the jet width is $R e=\frac{\langle u\rangle \delta}{\nu} \sim 70000$. At this location, the different characteristic scales of the jet are the integral scale $L \sim 55 \mathrm{~mm}$ (based on the jet's half-width), the Taylor micro-scale $\lambda \sim 3.5 \mathrm{~mm}$ and the Kolmogorov scale $\eta \sim 0.1 \mathrm{~mm}$. All the disks we use are smaller than the integral scale of the flow and much larger than either the dissipative scale or the Taylor micro-scale.

Force fluctuations are performed using a highly sensitive quartz force sensor of type 9203 from Kistler measuring compressive force from few $\mathrm{mN}$ to $500 \mathrm{~N}$. The sensor is mounted on the disk in order to be sensitive to the force component that is parallel to the main flow direction $u$. This component corresponds to the drag exerted on the disk (see fig. 1). As we need a high-resolution frequency of the drag fluctuations, particular attention has been given to the measurement itself. Indeed, fluctuations of drag are so weak especially at high frequencies, that any vibrations of the mechanical structure of the sensor support will considerably mask the true aerodynamic drag. Many different materials were tried for the holding stand until the force sensor was directly placed on a block of dough whose property is to be exclusively plastic (non-elastic) and the disk was stuck to the force sensor with bee wax. There is no screw, no mechanical articulation in our system. This special setup has allowed
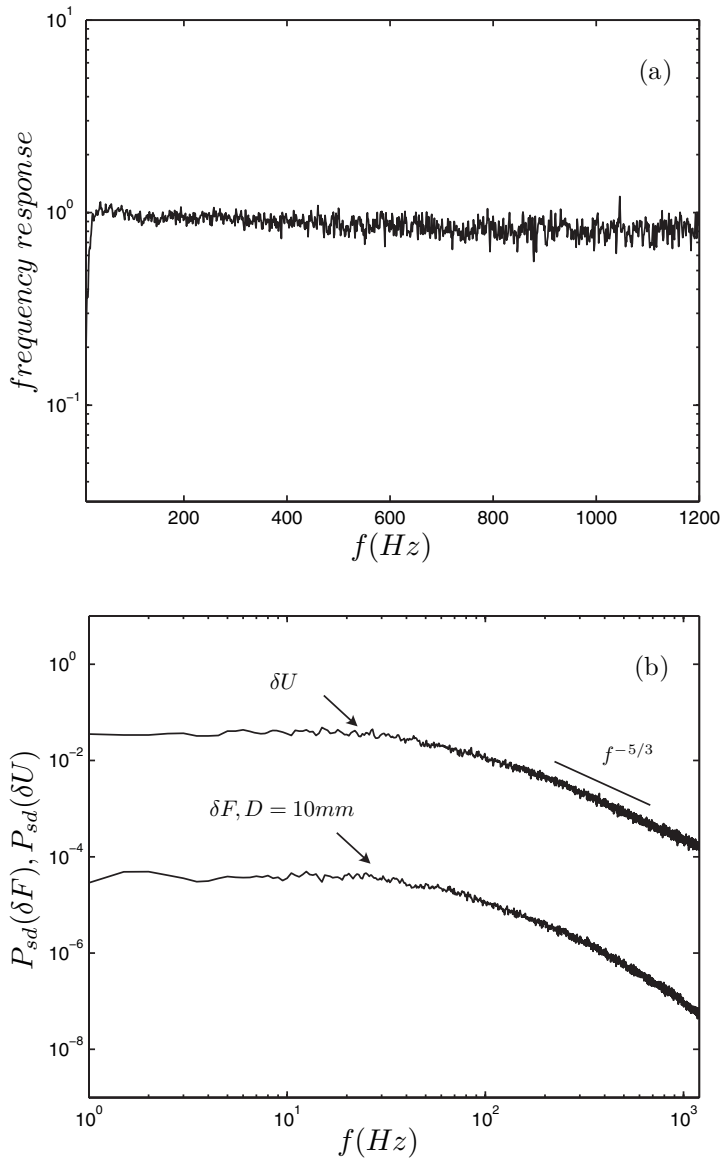

Fig. 2: (a) Frequency response of the force transducer measured as explained in text while the transducer is loaded with the $D=40 \mathrm{~mm}$ disk. (b) Comparison of power spectra of local velocity measurement (hot wire) and drag fluctuations of the disk of diameter $D=10 \mathrm{~mm}$.

us to push away all the resonances, which of course still exist, as far as we can. From this particular setup, the frequency response is directly measured using an electric coil supplied with a white noise current and placed in front of the disk. The random electromagnetic force suffered by the magnet is then compared to the initial white noise excitation. Figure 2(a) displays the frequency response obtained with the disk of diameter $D=40 \mathrm{~mm}$. From this result, we can obtain reliable measurements of the force fluctuations up to $1200 \mathrm{~Hz}$. For comparison in the work [10], it is the response of a flexion that is measured and the cut-off is much lower about $30 \mathrm{~Hz}$. On the other hand, our transducer is not sensitive to the low-frequency fluctuations, the corresponding high-pass filtering is about $10 \mathrm{~Hz}$. In the following, we shall call $\delta F(t)$ the drag fluctuations.

A hot-wire probe is used to obtain a local kinetic energy. The measurement point is localized on the jet axis, $10 \mathrm{~mm}$ upstream from the disk as displayed in fig. 1(a). The hot wire is always removed when drag is measured to avoid any contaminations. The hot wire is placed in such a way to 
be sensitive to $\sqrt{u(t)^{2}+v(t)^{2}}$ which is the modulus of the velocity projected in a plane containing the jet axis and the measurement point. We denote by $\epsilon(t)=\frac{1}{2}\left(u(t)^{2}+v(t)^{2}\right)$ the quantity corresponding to a local kinetic energy and by $\delta \epsilon(t)$ its fluctuation about the mean.

Finally, we perform Particle Image Velocimetry measurements (using a LAVISION PIV setup) to extract the instantaneous velocity field whose components are $u$ and $v$ (fig. 1(b)). The light sheet is produced by two lasers Nd: Yag of $50 \mathrm{~mJ}$ per pulse. A 12-bit camera with a CCD resolution of $1200 \times 1600$ is used. The seeding is insured by an olive oil spray placed at the entrance of the fan that generates the jet. The measurements area covered by the camera field is $80 \mathrm{~mm} \times 60 \mathrm{~mm}$ and the corresponding image resolution is $0.05 \mathrm{~mm}$ per pixel. For the inter-correlation postprocessing of the images, we use interrogation windows of 16 pixels $\times 16$ pixels. The resolution for the velocity measurements is then $0.8 \mathrm{~mm}$. The square area of dimensions $D \times D$ denoted as the "averaged area" in fig. 1(b) is the surface on which spatial averaging will be performed (see later in the text). In the following, symbols $\langle\ldots\rangle$ denotes temporal averaging. During a PIV acquisition, the sampling frequency of the velocity field is not constant in time and may fluctuate between $3 \mathrm{~Hz}$ and $10 \mathrm{~Hz}$. The PIV setup does not solve the dynamic of the large-scale structures since their typical turnover time is about $\frac{L}{U} \approx 0.05 \mathrm{~s}$. Temporal statistics are performed over 2000 velocity fields that can reasonably be assumed as independent.

Results. - We first use the smallest disk $(D=10 \mathrm{~mm})$. We measure the fluctuating drag, $\delta F(t)$ and the local velocity with the hot wire $\sqrt{u(t)^{2}+v(t)^{2}}$ located upstream from the disk (see fig. 1(a)). Figure 2(b) shows the comparison between power spectra of the local velocity (which correspond to a kinetic-energy spectrum) and drag measurements. The kinetic-energy spectrum falls as the classical $f^{-5 / 3}$ law as expected in the Kolmogorov theory. The power spectrum of the drag fluctuations is very similar to that of the local kinetic-energy spectrum in the range of the low frequencies. A different behavior is observed for the high-frequencies range where the drag fluctuation power spectrum decreases more abruptly than the kinetic-energy spectrum.

In fig. 3, the Probability density function (Pdf) of the reduced drag fluctuation $\delta F^{\prime}$ obtained for the smallest disk $D=10 \mathrm{~mm}$ is compared to the Pdf of the local kineticenergy fluctuations $\delta \epsilon^{\prime}$ measured from the local velocity probe. Both Pdfs are also very similar, especially the tail for positive events that superimposes satisfactorily. The Pdf asymmetry of the kinetic energy is at first order a consequence of the quadratic non-linear deformation of the velocity Pdf which is mainly Gaussian. This is confirmed by the good superimposition on the same graph of a Chi2 distribution. Although the asymmetry sign is in agreement with the previous result of Ciliberto et al. [10] for drag statistics, we do not observe exponential tails as they do.

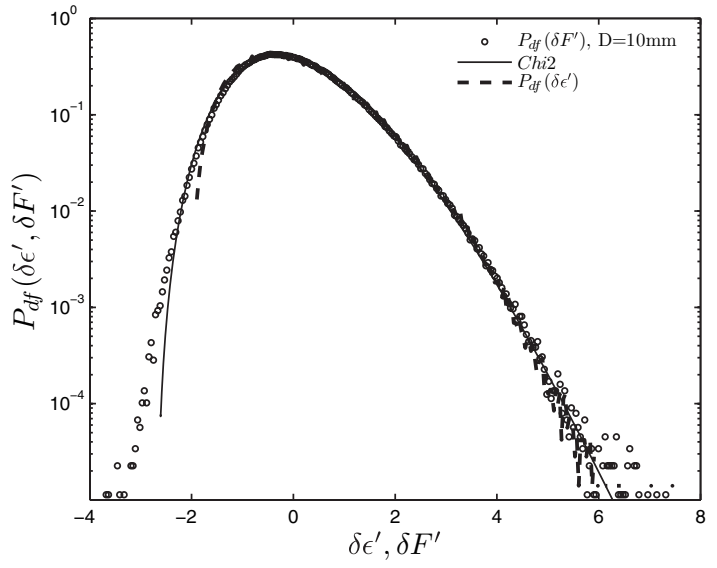

Fig. 3: Comparison of the Pdf of the reduced drag fluctuations $\delta F^{\prime}=\frac{\delta F}{\sqrt{\left\langle(\delta F)^{2}\right\rangle}}$ obtained from the disk of diameter $D=10 \mathrm{~mm}$ with the Pdf of the reduced local kinetic-energy fluctuations $\delta \epsilon^{\prime}=\frac{\delta \epsilon}{\sqrt{\left\langle(\delta \epsilon)^{2}\right\rangle}}$ obtained from the local hot-wire measurements and a Chi2 distribution.

This difference is related to the important role that plays the large-scale flow inhomogeneity: our disks are placed on the jet axis, while in Ciliberto et al. [10] the disk is placed at a distance of four disk's diameter off the jet axis. From the spectra in fig. 2(b) and the Pdfs in fig. 3, we find that the drag fluctuations behave, at first order as the fluctuations of the local kinetic energy in the jet. The result is consistent with the assumption that the drag fluctuations are simply associated with the pressure head fluctuations on the disk. In order to check this idea, we placed the disk in a uniform flow (wind tunnel of $400 \mathrm{~mm} \times 400 \mathrm{~mm}$ in test section) having the same velocity say, $10 \mathrm{~m} / \mathrm{s}$. In this case the drag fluctuation are too small to be measurable. We then conclude that drag fluctuations due to the wake are negligible compared to that of the forehead disk when the incoming flow is the turbulent jet. We can establish a simple relationship, based on dimensional arguments, between the head pressure and the kinetic energy. For a uniform incoming incompressible fluid of velocity $U$, the pressure head on the disk is given by the Bernoulli's theorem, $P=P_{0}+\frac{1}{2} \rho U^{2}$, with $P_{0}$ the free-stream pressure and $\rho$ the air density. Replacing $U$ by $u(t)$, we obtain that the pressure head fluctuations, and hence the drag fluctuations in our case, depends on the kinetic-energy fluctuations in the bulk upstream from the disk. This is exactly what is revealed by fig. 3. Assuming that the velocity fluctuations are small compared to the mean velocity in the jet, one obtains that $\delta P(t) \sim \rho\langle u\rangle \delta u(t)$. In this case, fluctuations of the local velocity are proportional to the drag fluctuations. This dimensional relation explains why we observe similar spectra in fig. 2(b). Of course Bernoulli's theorem does not hold rigorously in our case, our result only expresses a conservation law between pressure and kinetic-energy variation. In the detail the spectrum of the local velocity in fig. 2(b) decreases more abruptly at high 

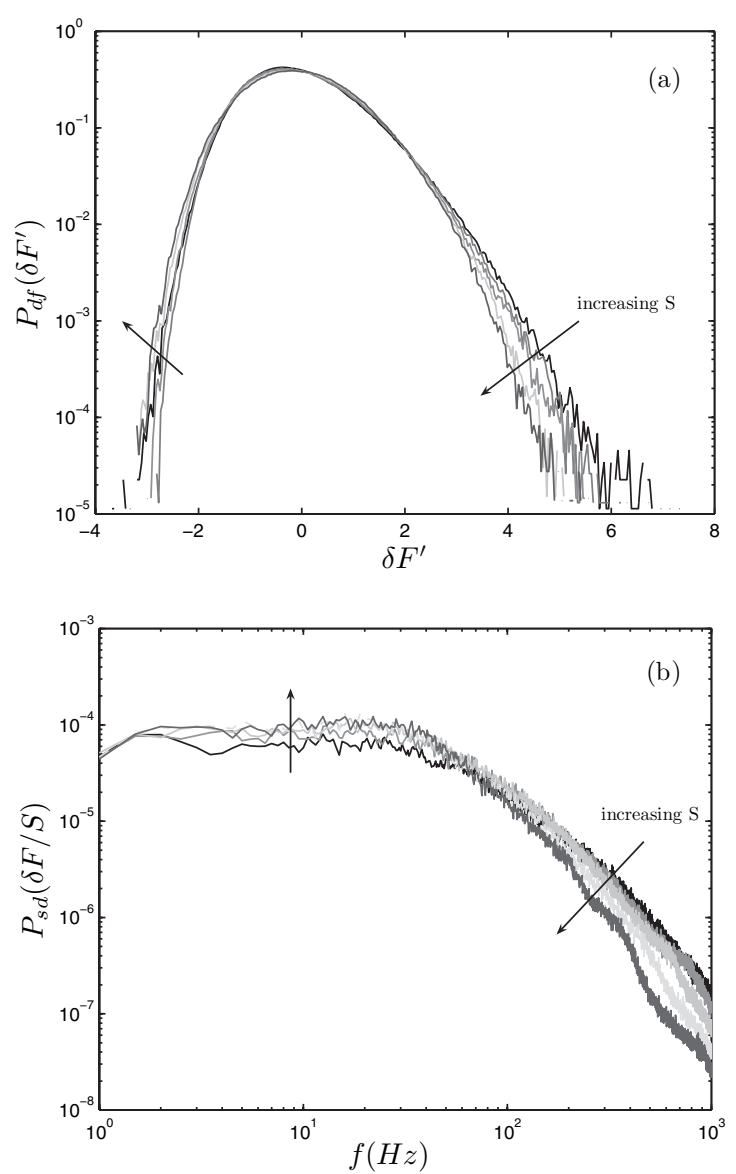

Fig. 4: (a) Evolution of the Pdfs of the reduced drag fluctuations $\delta F^{\prime}=\frac{\delta F}{\sqrt{\left\langle(\delta F)^{2}\right\rangle}}$ as a function of $S$. (b) Evolution of normalized drag spectra (each power spectrum has been divided by the corresponding disk surface) as a function of $S$. The arrows indicate the curves legend (see text).

frequencies than that of the drag fluctuations. This faster decrease is related to a spatial averaging effect. The effect is confirmed in the following when the size of the disk is increased.

The magnitude of the disk surface $S$ influences the statistics of the drag fluctuations. The Pdfs of the drag in fig. 4(a) tend to symmetrize as $S$ increases: the tail at large positive drag fluctuations is shortened while the other tail for the negative fluctuations is reinforced (this observation is displayed by arrows in fig. 4(a)). The effect is weak but very significant since the hierarchy of the tails follows the hierarchy of the disk diameters. While the Chi2 distribution represents well the Pdf for $D=$ $10 \mathrm{~mm}$ (fig. 3), it becomes less and less accurate as $D$ increases. In fig. $5(\mathrm{a})$, we plot the skewness $\left\langle\delta F^{\prime 3}\right\rangle v s$. $S / S_{L}$, where $S_{L}=\pi\left(\frac{L}{2}\right)^{2}$ is the characteristic surface of the integral scale impacting the disk defined from $L=$ $55 \mathrm{~mm}$, the half-width of the free jet at the disk location. We can see that the skewness decreases continuously as the disk surface increases. The spatial averaging effect is also clearly visible in fig. 4(b) that shows the evolution
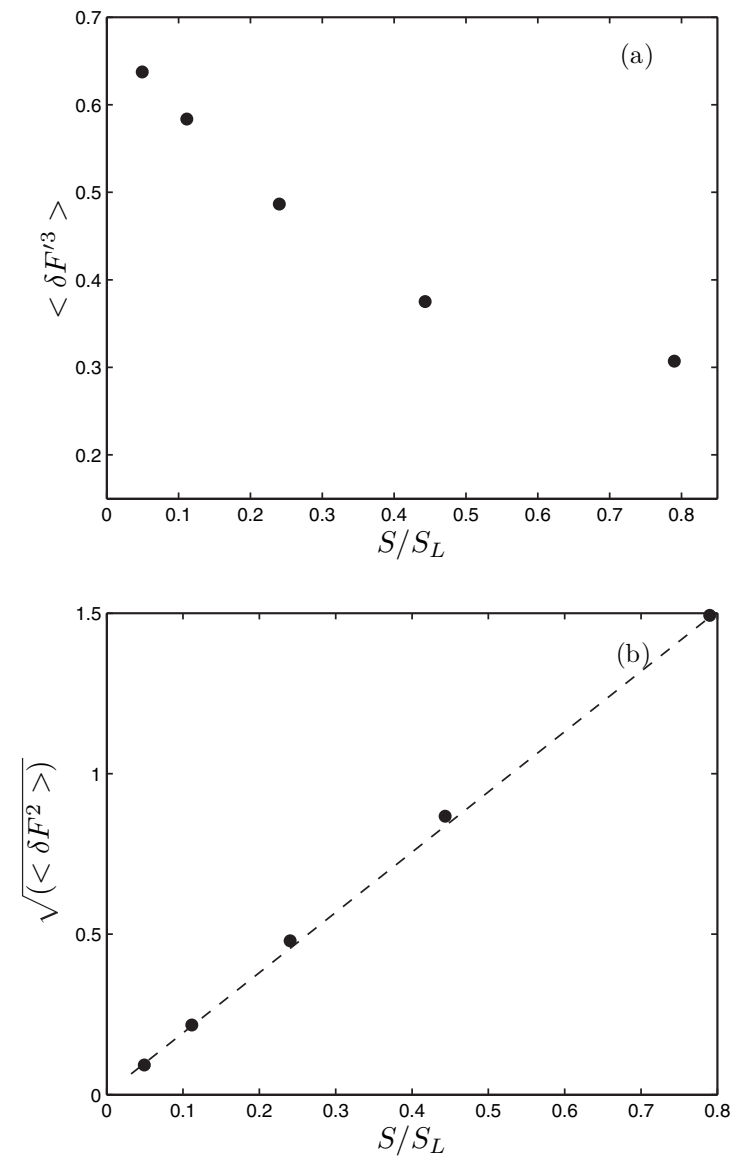

Fig. 5: (a) Skewness of drag fluctuations vs. the disk surface $S / S_{L}$. (b) Standard deviation vs. the disk surface $S / S_{L}$. The dashed line is a linear law.

of the power spectra of drag fluctuations with $D$. Each power spectrum has been divided by the corresponding disk surface. A cut-off frequency due to the size of the integration surface shifts to lower values as $D$ increases. As the integration surface increases, scales smaller than the integration surface naturally average and some energy at high frequencies is then lost. While more energy related to the small scales is lost as $S$ increases, more energy is created in the large scales since the plateau at low frequencies is elevated (the effect is displayed by arrows in fig. 4(b)). On the other hand, we find that the root-meansquare (rms) value $\sqrt{\left\langle\delta F^{\prime 2}\right\rangle}$ is linear with the surface in fig. $5(\mathrm{~b})$ or equivalently that $\sqrt{\left\langle\delta F^{\prime 2}\right\rangle}$ divided by the surface is almost constant in fig. 6(b) (squares). In consequence of the Parseval relation, all the spectra in fig. 4(b) have the same integral over the whole frequencies range. The spatial averaging due to the disk size does not smooth the fluctuations as it would be expected from the central limit theorem. The reason should be the interaction between the disk and the jet that is observed as a redistribution of the high-frequencies energy (small scales) to the low-frequencies energy (large scales) by keeping constant the total energy of the fluctuations per 

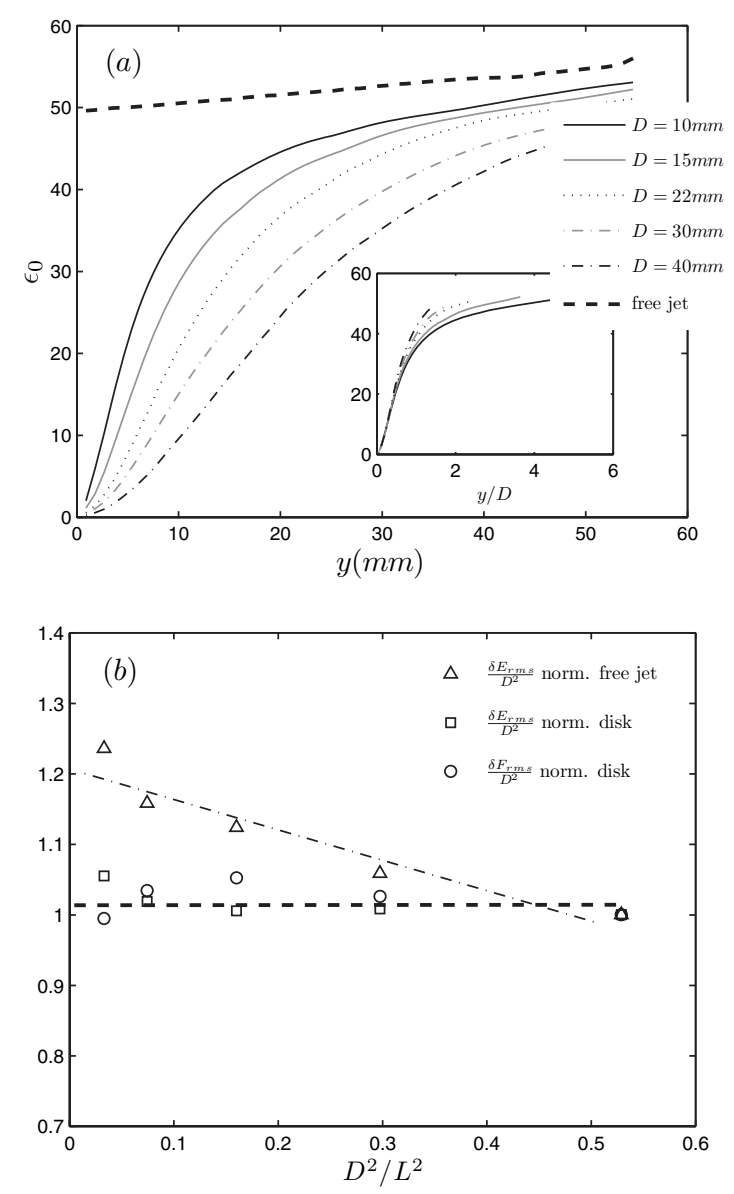

Fig. 6: (a) Kinetic energy of the mean flow on the jet axis $(x=0)$ vs. the distance to the disk $y$. Inset: same data but plotted vs. $y / D$. (b) Rate of fluctuations of the kinetic energy contained in a $D \times D$ area vs. $D^{2} / L^{2}$. The rate of fluctuations of the free jet (triangles) is compared to a model of correlated degrees of freedom having a correlation length of $L_{C}=\frac{2}{3} L \simeq$ $37 \mathrm{~mm}$ (continuous line, see text). The rate of fluctuations in front of the disks (circles) is compared to the density of the rms fluctuations about the mean of the drag exerted on the disk presented in fig. 5(b) (squares).

surface unit. The jet-disk interaction strongly modifies the turbulent mean flow by imposing a turbulent boundary layer on the disk. In fig. 6(a), the kinetic energy of the mean flow extracted from PIV measurements along the jet axis, $\epsilon_{0}(0, y)=\frac{1}{2}\left(\langle u\rangle^{2}+\langle v\rangle^{2}\right)$ decreases toward zero on the disk. As shown in the inset of fig. 6(a), for every disk, the decreasing laws superimpose when $y$ is scaled by $D$. The typical length scale for the turbulent boundary layer on each disk is then simply given by $D$.

The last global variable we have considered is extracted from the PIV measurements. It is a total kinetic energy $E$ contained in a square area of the $x y$-plane whose size is equal to the disk diameter $D$ (see fig. $1(\mathrm{~b})$ ) and defined as

$$
E(t)=\sum_{i, j \in D \times D}\left(u_{i, j}(t)^{2}+v_{i, j}(t)^{2}\right)
$$

where $(i, j)$ denotes the location of the velocity vector inside the area $D \times D$. The local velocities measured with the PIV setup already result from a spatial averaging over a square area of size $\delta s=0.08 \mathrm{~mm} \times 0.08 \mathrm{~mm}$. It corresponds to the size of the interrogation window and also to the mesh size of the location matrix. For each disk, we acquired 2000 velocity fields and then obtained 2000 realizations for $E$. We also perform the same velocity measurements without disk in order to characterize the averaging effect of the free jet. In this case, we also consider the five averaged areas corresponding to the five disk diameters. For each realization set of $E$, we computed the rate of fluctuations defined as: $\frac{\delta E_{r m s}}{D^{2}}$, where $\delta E_{r m s}=$ $\sqrt{\left\langle(E-\langle E\rangle)^{2}\right\rangle}$. This quantity is plotted in fig. 6(b) vs. the surface of integration $D^{2} / L^{2}$ for the free jet and jet with disks. The quantity is normalized by its magnitude for the larger area of integration, say $D=40 \mathrm{~mm}$ (for which $D^{2} / L^{2}=0.53$ ). The free jet exhibits a pronounced averaging effect, the larger the size of integration the smaller the rms fluctuation rate.

Discussion and conclusion. - The aim of this work was to study the consequence of the spatial averaging of turbulence scales on the drag fluctuations of a disk placed in a turbulent jet. The equivalence between the local kinetic energy in front of the disk and the drag is shown with the smaller disk $(D=10 \mathrm{~mm})$ by the similarities of the Pdf (fig. 3) and the spectra (fig. 4). When the disk size is increased, the rms fluctuations density of the global kinetic energy computed in an area in front of the disk behave mostly as the rms fluctuations density of the drag (fig. 6(b)). We can then assume that the drag fluctuations exerted on the disk are mainly governed by the global kinetic energy contained in an area in front of the disk. In other word, the way the drag statistics behave as the disk size $D$ increases should be similar to that of the statistics of the global kinetic energy taken over an area of size $D$ in front of the disk.

For the free jet, the rms density fluctuations of the global kinetic energy contained in a box decreases continuously as the size of the box increases (fig. 6(b)). This smoothing of the fluctuations is consistent with a constant correlation length, characteristic to the free jet (proportional to the large-scale length), and smaller than the box sizes. It is equivalent to an increase in the effective number of degrees of freedom as the box size is increased.

When a disk is introduced in the turbulent jet, the smoothing effect of the rms density fluctuations averaging nearly disappears (fig. 6(b)). Actually the presence of the disk in the jet modifies the turbulent flow property in front of the disk compared to that of the free jet. A new correlation length, corresponding to the boundary layer thickness on the disk is introduced. We find this length to be proportional to the disk diameter $D$ (fig. 6(a)), which may be consistent with a constant number of degrees of freedom irrespective of the disk diameter. Although we do not observe spatial averaging effect on the rms density 
fluctuations of the drag, the low-pass filtering of the spectra (fig. 4(b)) and the Pdfs symmetrization (fig. 4(a)) indicate, on the other hand, a significant disk's size effect. This low-pass filtering is actually associated with a redistribution of the high-frequency energy fluctuations to the low frequencies. In conclusion, increasing the body size in a turbulent jet will not lead to a reduction of its drag fluctuation rate. The reason is that the body imposes a new correlation length proportional to its size which acts on the drag as a low-pass filter and symmetrizes the PDF, but keeps the fluctuation rate constant.

The authors would like to thank A. Noullez for fruitful discussions. This work was supported by the ANR Project No. 06-BLAN-0363-01 "HighSpeedPIV".

\section{REFERENCES}

[1] Labbé R., Pinton J.-F. and Fauve S., J. Phys. II, 6 (1099) 1996.

[2] Titon J. H. and Cadot O., Phys. Fluids, 15 (2003) 625.

[3] Cadot O. and Titon J. H., Phys. Fluids, 16 (2004) 2140.

[4] Schumacher J. and Eckhardt B., Physica D, 187 (2004) 370.

[5] Titon J. H. and Cadot O., Eur. Phys. J. B, 45 (2005) 289.

[6] Aumaître S., Fauve S., McNamara S. and Poggi P., Eur. Phys. J. B, 19 (2001) 449.

[7] Aumaître S., Fauve S. and Pinton J.-F., Eur. Phys. J. B, 16 (2000) 563.

[8] Pumir A., Phys. Fluids, 8 (1996) 3112.

[9] Noullez A. and Pinton J.-F., Eur. Phys. J. B, 28 (2002) 231.

[10] Ciliberto S., Garnier N., Hernandez S., Lacpatia C., Pinton J.-F. and Ruiz Chavarria G., Physica A, 340 (2004) 240. 